\section{AB0790 EVALUATING THE EFFECTIVENESS OF INTRA- ARTICULAR KNEE INJECTION USING ALLOGENIC PLATELET DERIVED LYOPHILIZED GROWTH FACTORS IN EGYPTIAN PATIENTS WITH SYMPTOMATIC PRIMARY KNEE OSTEOARTHRITIS}

${ }^{1}$ Rasmia Elgohary ${ }^{*}$ Amany Diab ${ }^{1}$, Hala Elgndy ${ }^{1}$, Hossam Fahmy ${ }^{2}$, Kamel Gado ${ }^{1}$. ${ }^{1}$ Kasr Alainy School of Medicine, Cairo University, Egypt, Internal Medicine, cairo, Egypt, ${ }^{2}$ Faculty of Medicine, Ain Shams University, Clinical Pathology, cairo, Egypt

Background: Using intra-articular injections of autologous platelet-rich plasma (PRP) has showen promising results in treatment of knee osteoarthritis (KOA). Its regenerative properties depends on the amount of growth factors (GF) released after platelets activation. Using large-volume of blood for obtaining allogenic PRP can provide higher concentrations of platelet and GF. However once prepared, PRP is stable for only 8-hours. Freeze drying (lyophilization) can be used to stabilize the biological materials for prolonged storage without causing their damage (1).

Objectives: Investigating the effectiveness of intra-articular allogenic lyophilized growth factors (L-GF) injection in patients with symptomatic KOA using clinical and knee ultrasonography assessment.

Methods: 30-patients with symtomatic primary KOA, diagnosed according to revised criteria of the ACR (2), were enrolled. Local ethical approval was obtained. All patients had given a written informed consent. The study group was equally randomized into intervention and control groups. Both was subjected to baseline functional assessment using WOMAC score; evaluating pain, stiffness, and physical function (3), and ultrasonography (US) assessment of knee effusion. The patients in control group were kept on their medications without intervention. The intervention group was recieved two doses of L-GF; at baseline and after 2-months. L-GF was prepared from platelets derived from individual whole blood donations. Each unit of platelets was tested in and found to be non-reactive for HBsAg, HIV I \& II antibodies, HIV p-24 antigen, HCV antibodies and antibodies to Treponema Palidum, by licensed assay methods. Seronegative plasma were further examined by Nuclear Acid Testing. Further viral inactivation by UV-radiation and Riboflavin by the Mirasol system was done. The platelets in the buffy coat layer were activated using $\mathrm{CaCl}_{2}$ to release their GF. Excess water, cellular elements and fibrinogen were removed and the remaining GF were "ultraconcentrated." Lyophilization of the obtained GF was then processed. The L-GF was supplied as powder in tightly sealed container and was stored at $2^{\circ}$ to $8^{\circ} \mathrm{C}$. Prior to use, reconstitution of the product was done using 1-ml saline and 1-ml lignocaine then the mixture was kept at ambient temperature for 5-min. to ensure complete protein re-hydration. After 6-months the WOMAC score and knee US were repeated for all particepients. Then percent of improvement of WOMAC and US detected effusion were calculated for all patients.

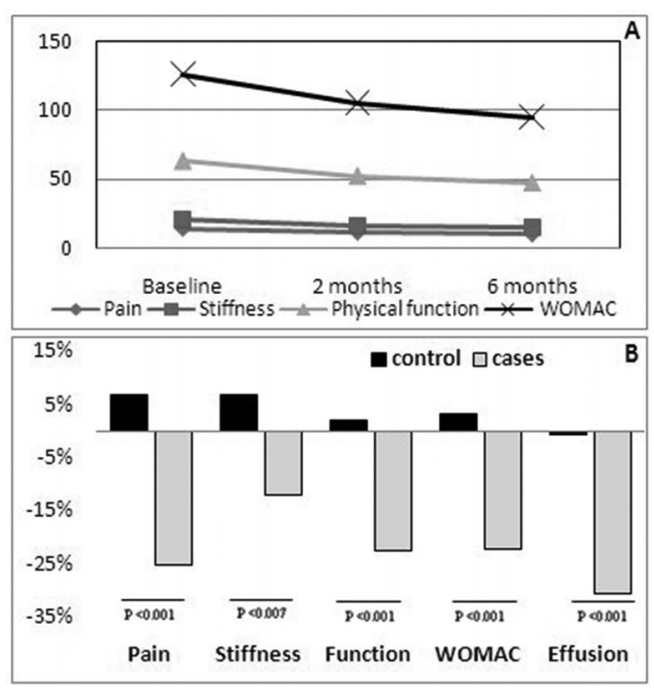

Results: The patients in the intervention group was significantly older (56 \pm 9 years) and had heigher BMl $\left(38 \pm 5.3 \mathrm{~kg} / \mathrm{m}^{2}\right)$ in comparison to control $\left(44 \pm 11\right.$ years, $\left.33 \pm 5.6 \mathrm{~kg} / \mathrm{m}^{2}\right)$. Both groups showed statistically insignificant difference regarding the baseline total WOMAC score and radiographic assessment. Post injection pain was reported by all patients in intervention group, it lasted only for $2.4 \pm 0.83$ hours and was graded as mild. The mean of total WOMAC score and its 3-compenents in addition to knee effusion were decreased significantly in the intervention group after 2 months and 6 months of follow-up (figuer-A). After 6-months, the intervention group showed statistically significant persentage of improvement regarding WOMAC scores and effusion in comparison to the control (figuer-B).

Conclusion: Allogenic L-GF was well tolerated and showed encouraging results in patients with symptomatic KOA, regarding improvement of pain, stiffness and function in addition to decreasing knee effsuion.

\section{REFERENCES}

[1] Pan L, et al. Aesth Plast Surg. 2016;40: 157.

[2] Altman R, et al. Arthritis Rheum 29:1039, 1986

[3] Bellamy N, et al. J Rheumatol 1988; 15:1833-40.

Disclosure of Interests: Rasmia Elgohary: None declared, Amany Diab: None declared, Hala Elgndy: None declared, Hossam Fahmy: None declared, Kamel Gado Speakers bureau: have been paid as a speaker to pharmaceutical companies (Novartis, EVA pharm) and also to attend their advisory board

DOI: 10.1136/annrheumdis-2019-eular.1978

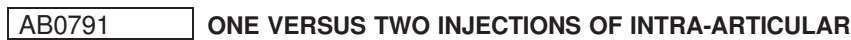 HIGHLY CROSS-LINKED HYALURONAN YEARLY IN PATIENTS WITH KNEE OSTEOARTHRITIS: INSIGHTS FROM ROUTINE CLINICAL PRACTICE}

Tsvetoslav Georgiev, Rumen Stoilov. University Hospital "St. Ivan Rilski" - Sofia, Clinic of Rheumatology, Sofia, Bulgaria

Background: Hyaluronic acid $(\mathrm{HA})$ is a natural polysaccharide, which is an important structural component of synovial fluid and cartilage. There are different injectable forms of HA used for clinical application. Highly cross-linked/high-molecular-weight hyaluronans (HMWHA) provide additional stability and improve functionality [1], resulting in longer bioavailability in the knee joint and promoting viscoinduction [2]. A direct comparison between the two most common injection protocols of HMWHA in our routine practice is of great practical interest, since viscosupplementation is among the most commonly used treatment modalities for patients with knee osteoarthritis (KOA) in Bulgaria [3]

Objectives: Our aim was to compare the clinical effectiveness of two different regimens for injecting intra-articular HMWHA in KOA patients under 'real-world' conditions in routine clinical practice.

Methods: This prospective, open-label, observational study included 50 patients with KOA who were followed for a period of 1 year. They were divided into two therapeutic arms according to their preselected treatment regimen: patients injected once with HMWHA $(n=25$; group 1$)$ at baseline and patients injected twice 6 months apart with HMWHA $(n=25$; group 2). A $100-\mathrm{mm}$ visual analogue scale (VAS) for pain, disease-specific (Western Ontario \& McMaster Universities Osteoarthritis Index [WOMAC]) and generic (health assessment questionnaire - disability index [HAQ-DI]) questionnaires were used to evaluate patients at baseline, three months, six months, and one year later. Standardized radiographs were obtained at baseline and after one year. The response to the treatment was determined using the OMERACT-OARSI set of responder criteria [4]. Results: A single injection of HMWHA resulted in a statistically significant improvement in pain even at 12 months $(\Delta V A S=10.12 \pm 14.5 \mathrm{~mm}, \mathrm{p}$ $<0.05$ ), although the effect progressively decreased after the third month when $\triangle$ VAS was $18 \pm 14.31 \mathrm{~mm}, p<0.05$ (Figure 1). If the two regimens of HA injections were directly compared, the mean difference of pain improvement for the year of follow-up was about $9 \mathrm{~mm}$ in favor of group 2, but this result was not "statistically" significant (10.12 vs 19.16 $\mathrm{mm}, \mathrm{p}>0.05)$. Physical function was statistically improved in both 